\title{
Anticoagulant treatment in patients with pulmonary arterial hypertension associated with systemic sclerosis: More shadows than lights
} journals.sagepub.com/home/jso

\author{
Massimiliano Palazzini, Alessandra Manes, Enrico Gotti, Fabio Dardi, Andrea Rinaldi, Nazzareno Galiè
}

Department of Experimental, Diagnostic and Specialty Medicines (DIMES), University of Bologna, Bologna - Italy

\begin{abstract}
Pulmonary arterial hypertension is a chronic and progressive disease characterized by elevated pulmonary artery pressure and pulmonary vascular resistance leading to heart failure and premature death. Pulmonary arterial hypertension is characterized by proliferative and obstructive lesions in the distal pulmonary arteries and some descriptions include also thrombotic lesions. Despite this, in an era when multiple effective pulmonary arterial hypertension therapies are available, the role of anticoagulation in the treatment of pulmonary arterial hypertension remains uncertain. In particular, anticoagulant treatment in pulmonary arterial hypertension associated with connective tissue disease seems to be associated with unfavorable risk to benefit ratio due to an increased rate of bleeding from the gastrointestinal tract. However, anticoagulation may be required in conditions with increased thrombophilia like in the presence of lupus anticoagulant phenomenon or in the presence of anticardiolipin antibodies.
\end{abstract}

Keywords: Anticoagulation, Pulmonary arterial hypertension, Systemic sclerosis, Therapy

Pulmonary arterial hypertension (PAH) is a severe and chronic disease characterized by a progressive obstructive remodeling of the distal pulmonary arteries that results in increased pulmonary vascular resistance and pulmonary arterial pressure. PAH includes a heterogeneous group of clinical entities such as idiopathic PAH (IPAH), heritable PAH (HPAH), drug- and toxin-induced $\mathrm{PAH}$, and $\mathrm{PAH}$ associated with congenital heart disease, connective tissue disease (CTD), and HIV infection (1).

The exact processes that initiate the pathological changes seen in PAH are largely unknown. It is hypothesized that the interaction between constitutional predisposition and environmental risk factors may be involved in the initial stages

Accepted: December 13, 2017

\author{
Corresponding author: \\ Massimiliano Palazzini \\ Department of Experimental \\ Diagnostic and Specialty Medicine (DIMES) \\ University of Bologna \\ Via Massarenti, 9 \\ 40138 Bologna, Italy \\ massimiliano.palazzini@unibo.it
}

of the disease. However, the exact role of the autoantibodies in CTD or the viral involvement in HIV infection on the pathogenesis of associated PAH is unclear. It appears that a specific injury on the vessel wall of distal pulmonary arteries may initiate, in predisposed individuals, a pathobiological cascade of events leading to a common final pathological obstructive condition. Pathological lesions in PAH patients affect the distal pulmonary arteries $(<500 \mu \mathrm{m}$ of diameter) and are characterized by medial hypertrophy, intimal proliferative and fibrotic changes, adventitial thickening with moderate perivascular inflammatory infiltrates, complex lesions (plexiform and dilated), and also thrombotic lesions (2).

It is unclear whether thrombotic vascular lesions are an integral part of the pulmonary vascular pathology or are simply an epiphenomenon. In early pathological series, a high prevalence of vascular thrombotic lesions at postmortem examination were described in patients diagnosed as IPAH (3). However, it is uncertain whether the differential diagnosis with chronic thromboembolic pulmonary hypertension (CTEPH) was performed according to the modern criteria (1). There is some biological rationale to support the hypothesis that thrombotic processes are a significant pathophysiologic feature of $\mathrm{PAH}$ and that its evolution contributes to disease 
Table I - Recent studies of anticoagulation therapy in PAH

\begin{tabular}{|c|c|c|c|c|}
\hline Author & & Subjects & Exposure & Outcome \\
\hline \multirow[t]{2}{*}{ Olsson et al. (14) } & COMPERA registry & 1283 & $\begin{array}{l}\text { Vitamin } \mathrm{K} \text { antagonists, } \\
\text { heparins, and novel oral } \\
\text { anticoagulation }\end{array}$ & \\
\hline & & IPAH $(n=800)$ & & $\begin{array}{l}\text { Better } 3 \text {-year survival }(p=0.006) \text { in } \\
\text { patients on anticoagulation compared } \\
\text { with patients who never received } \\
\text { anticoagulation }\end{array}$ \\
\hline \multirow[t]{2}{*}{ Preston et al. (20) } & $\begin{array}{l}\text { REVEAL registry } \\
\text { (matched study) }\end{array}$ & 374 & Vitamin $\mathrm{K}$ antagonist & \\
\hline & & IPAH $(n=288)$ & & $\begin{array}{l}\text { No survival difference with warfarin } \\
\text { in IPAH patients in comparison with } \\
\text { matched controls. }\end{array}$ \\
\hline
\end{tabular}

IPAH=idiopathic pulmonary arterial hypertension; SSc=systemic sclerosis.

progression (4). However, these abnormalities apparently have not been described in the associated forms of $\mathrm{PAH}$, including CTD-PAH.

PAH is a common complication of systemic sclerosis (SSc), relatively uncommon in mixed CTD and systemic lupus erythematosus and rare in rheumatoid arthritis, Sjogren's syndrome, and inflammatory myositis (5). Patients with CTD-PAH, especially with PAH-SSc, have been included in many of the randomized controlled trials with drugs in $\mathrm{PAH}$, and usually, they represent the second largest group after IPAH. Several studies demonstrate that CTD-PAH patients have a worse prognosis as compared with other PAH subgroups and that patients with SSc-PAH have particularly poor outcomes (6). Nevertheless, the advanced PAH therapies determine improvements in clinical, haemodynamic, and exercise capacity measures as well as health-related quality-of-life outcomes, also in SSc-PAH patients compared with placebo $(7,8)$.

Oral anticoagulant treatment has been indicated for patients with IPAH, HPAH, and PAH due to anorexigens in different editions of the pulmonary hypertension $(\mathrm{PH})$ guidelines with variable degrees of recommendation. Supporting evidence and safety data, however, are limited. In fact, only eight observational studies (two prospective and six retrospective) have evaluated the effect of chronic anticoagulation on the outcome of patients with IPAH, and six of them reported apparently favorable outcomes (3, 9-15). In 2006, a qualitative systematic review on anticoagulation in PAH performed by Johnson concluded that the literature appears to support a treatment effect of anticoag- ulation therapy with warfarin on mortality in IPAH but requiring randomized clinical trials (16). In fact, none of the studies was blinded, randomized, and placebo-controlled. Second, the studies date to a time when there was no specific therapy for $\mathrm{PAH}$ (only empiric use of vasodilators) and the size of the sample was extremely poor. Benefit of oral anticoagulation in CTD-PAH is even less clear as this strategy has been extrapolated from the questionable data collected in IPAH patients (17).

A Bayesian analysis of observational data from a longitudinal cohort of patients with SSc-PAH or IPAH concluded that there is a low probability that warfarin improves survival in SSc-PAH and IPAH. Given the availability of multiple $\mathrm{PAH}$ therapies with evidence-based clinical benefits, the potential additional effect of warfarin to improve outcomes is unclear if any (18).

Recently, a comparative prospective registry-based study (COMPERA) apparently showed that IPAH anticoagulated patients had a better survival as compared to nonanticoagulated ones. No survival benefit was observed in patients with $\mathrm{PAH}$ associated with other conditions like CTD-PAH (14). In this population, there is a trend $(p=0.08)$ towards a worse outcome associated with anticoagulation (14). However, the analysis performed in this study is influenced by the "immortal time bias" and even the favorable effect observed in IPAH patients is questionable (19). In fact, the survival in this study was computed since the inclusion of the patients in the registry and not since the actual starting of the anticoagulant therapy. The time between 
these two time-points is defined "immortal time" because the patient cannot die before receiving the first dose of anticoagulant therapy. In the study analysis, the "immortal time" was considered as survival on anticoagulant therapy thus inappropriately providing this survival extension to the treated patients as compared with the non-anticoagulated ones (19). In the REVEAL registry analysis, this bias was avoided and no significant survival increase was observed in IPAH patients who started warfarin while in the registry. In addition, in SSc-PAH patients, long-term warfarin treatment was associated with worse survival as compared with patients not receiving warfarin even after adjusting for confounders (20). Immortal time bias was avoided also in the comparison of PAH patients with or without anticoagulant treatment enrolled in the SERAPHIN trial, and no difference in the outcome was observed in patients not on anticoagulant therapy compared with those on anticoagulant therapy between IPAH and non-IPAH patients (IPAH - hazard ratio (HR): $0.97,0.72-1.32$ vs non-IPAH-HR: $0.71,0.49-1.02 ; p=0.19$ ) (21). Newest studies evaluating a role for anticoagulation in PAH, with mortality as an end point, are summarized in Tab. I. The detrimental effect of long-term warfarin in CTD-PAH patients may be explained by comorbidities. In fact, there is increased risk of bleeding from gastrointestinal telangiectasias or arteriovenous malformations in SSc-associated PAH patients as compared to IPAH or CTEPH patients $(22,23)$. In fact, in the retrospective analysis of PAH patients treated in the VU Medical Center hospital of Amsterdam, bleeding event rates with vitamin $\mathrm{K}$ antagonist drugs were 5.4 per 100 patient-years in patients with IPAH, 19 per 100 patient-years in CTD-PAH patients, and 2.4 per 100 patientyears in CTEPH patients (23). Major bleedings were independent of age, sex, target international normalized ratio (INR) range, documented INR actual value, vitamin $\mathrm{K}$ antagonist type, or right atrial pressure, but was associated with the concomitant use of prostacyclin analogues (23). In fact, the concurrent use of PAH-specific therapies with antiplatelet effect such as prostacyclin analogues may increase the risk of bleeding for the combined inhibition on multiple factors of the coagulation cascade.

A randomized controlled trial with the new oral anticoagulant apixaban in SSc-PAH patients (SPHInX registration number ACTRN12614000418673) is ongoing and may provide us with long awaited information on this treatment approach. In fact, this is the first clinical trial ever to evaluate the efficacy, safety, and cost-effectiveness of anticoagulant therapy in addition to approved PAH drugs in SSc-PAH patients (24).

In conclusion, the use of anticoagulant treatment remains controversial in patients with PAH and may be detrimental in the majority of patients with CTD-PAH. Anticoagulant treatment may be considered in conditions with $\mathrm{PH}$ and increased thrombophilia like in the presence of lupus anticoagulant phenomenon or in the presence of anticardiolipin antibodies.

\section{Disclosures}

Financial support: No grants or funding have been received for this study.

Conflict of interest: None of the authors has financial interest related to this study to disclose.

\section{References}

1. Galiè N and Humbert M. 2015 ESC/ERS Guidelines for the diagnosis and treatment of pulmonary hypertension: The Joint Task Force for the Diagnosis and Treatment of Pulmonary Hypertension of the European Society of Cardiology (ESC) and the European Respiratory Society (ERS): Endorsed by: Association for European Paediatric and Congenital Cardiology (AEPC), International Society for Heart and Lung Transplantation (ISHLT). Eur Heart J. 2016;37(1):67-119.

2. Dorfmüller $P$ and Humbert $M$. Progress in pulmonary arterial hypertension pathology: relighting a torch inside the tunnel. Am J Respir Crit Care Med. 2012;186(3):210-212.

3. Fuster V, Steele PM, Edwards WD, et al. Primary pulmonary hypertension: natural history and the importance of thrombosis. Circulation. 1984;70(4):580-87.

4. Johnson SR, Granton JT and Mehta S. Thrombotic arteriopathy and anticoagulation in pulmonary hypertension. Chest. 2006;130(2):545-552.

5. Humbert $M$, Sitbon $O$, Chaouat $A$, et al. Pulmonary arterial hypertension in France: results from a national registry. Am J Respir Crit Care Med. 2006;173(9):1023-1030.

6. Galiè N, Manes A, Farahani KV, et al. Pulmonary arterial hypertension associated to connective tissue diseases. Lupus. 2005;14(9):713-717.

7. McLaughlin V, Humbert M, Coghlan G, et al. Pulmonary arterial hypertension: the most devastating vascular complication of systemic sclerosis. Rheumatology. 2009;48(suppl 3):iii25-iii31.

8. Barst R. How has epoprostenol changed the outcome for patients with pulmonary arterial hypertension? Int J Clin Pract Suppl. 2010;64(168):23-32.

9. Rich S, Kaufmann E and Levy PS. The effect of high doses of calcium-channel blockers on survival in primary pulmonary hypertension. N Engl J Med. 1992;327(2):76-81.

10. Frank $\mathrm{H}$, Mlczoch J, Huber $\mathrm{K}$, et al. The effect of anticoagulant therapy in primary and anorectic drug-induced pulmonary hypertension. Chest. 1997;112(3):714-721.

11. Ogata $M$, Ohe $M$, Shirato $K$, et al. Effects of a combination therapy of anticoagulant and vasodilator on the long-term prognosis of primary pulmonary hypertension. Jpn Circ J. 1993;57(1):63-69.

12. Kawut SM, Horn EM, Berekashvili KK, et al. New predictors of outcome in idiopathic pulmonary arterial hypertension. Am J Cardiol. 2005;95(2):199-203.

13. Storstein $\mathrm{O}$, Efskind L, Müller $\mathrm{C}$, et al. Primary pulmonary hypertension with emphasis on its etiology and treatment. Acta Med Scand. 1966;179(2):197-212.

14. Olsson KM, Delcroix M, Ghofrani HA, et al. Anticoagulation and survival in pulmonary arterial hypertension clinical perspective. Circulation. 2014;129(1):57-65.

15. Hoeper MM, Sosada M and Fabel H. Plasma coagulation profiles in patients with severe primary pulmonary hypertension. Eur Respir J. 1998;12(6):1446-1449. 
16. Johnson SR, Mehta S and Granton JT. Anticoagulation in pulmonary arterial hypertension: a qualitative systematic review. Eur Respir J. 2006;28(5):999-1004.

17. Galiè N, Corris PA, Frost A, et al. Updated treatment algorithm of pulmonary arterial hypertension. J Am Coll Cardiol. 2013;62(25 suppl):D60-D72.

18. Johnson SR, Granton JT, Tomlinson GA, et al. Warfarin in systemic sclerosis-associated and idiopathic pulmonary arterial hypertension. A Bayesian approach to evaluating treatment for uncommon disease. J Rheumatol. 2012;39(2):276-285.

19. Suissa $S$ and Galié N. Letter by Suissa and Galié regarding article, Anticoagulation and survival in pulmonary arterial hypertension: results from the Comparative, Prospective Registry of Newly Initiated Therapies for Pulmonary Hypertension (COMPERA). Circulation. 2014;130(12):e107.

20. Preston IR, Roberts KE, Miller DP, et al. Effect of warfarin treatment on survival of patients with pulmonary arterial hypertension (PAH) in the registry to evaluate early and long-term PAH disease management (REVEAL). Circulation. 2015;132(25):2403-2411.

21. Galie N, Delcroix M, Ghofrani A, et al. Anticoagulant therapy does not influence long-term outcomes in patients with pulmonary arterial hypertension (PAH): insights from the randomised controlled SERAPHIN trial of macitentan. Eur Heart J. 2014(35):10.

22. Marie I, Antonietti M, Houivet E, et al. Gastrointestinal mucosal abnormalities using videocapsule endoscopy in systemic sclerosis. Aliment Pharmacol Ther. 2014;40(2):189-199.

23. Henkens IR, Hazenoot T, Boonstra A, et al. Major bleeding with vitamin $\mathrm{K}$ antagonist anticoagulants in pulmonary hypertension. Eur Respir J. 2013;41(4):872-878.

24. Calderone A, Stevens W, Prior D, et al. Multicentre randomised placebo-controlled trial of oral anticoagulation with apixaban in systemic sclerosis-related pulmonary arterial hypertension: the SPHInX study protocol. BMJ Open. 2016;6(12):e011028. 